\title{
Acceso y difusión de la producción científica iberoamericana; Biblioteca Virtual en Salud y Modelo de publicación electrónica SciELO
}

\author{
The Access and the Dissemination of Latin American Scientific \\ Production in Health Sciences; Virtual Health Library \\ and electronic publication model Scielo.
}

Jorge Veiga del Cabo, Ma Belén Jaén Casquero y Silvia Hernández Villegas

Biblioteca Nacional de Ciencias de la Salud. Instituto de Salud Carlos III.

Se describe el proyecto Biblioteca Virtual en Salud, sus orígenes y sus principales herramientas, y como estas contribuyen a facilitar el acceso y difusión de la producción científica iberoamericana en Ciencias de la Salud. Se hace hincapié en el modelo de publicación electrónica SciELO (Scientific Electronic Library Online), que recoge colecciones de revistas científico técnicas de calidad, en formato electrónico y de acceso gratuito. Se exponen con mayor detenimiento las herramientas que componen la Biblioteca Virtual en Salud de España: bases de datos bibliográficas (IBECS, MEDLINE, LILACS...), el proyecto "DeCS - Descriptores en Ciencias de la Salud", el Localizador de Información en Salud "LIS-España: Sitios Saludables" y el proyecto "Monografías y boletines a texto completo", entre otros.

Palabras clave: Biblioteca Virtual en Salud, SciELO, publicación electrónica, DeCS, LIS-España: Sitios Saludables
The article describes the Virtual Health Library's project as well as its origin and main tools, and how these tools contribute to facilitate the access and the dissemination of Latin American scientific production in Health Sciences. The article emphasizes the SciELO (Scientific Electronic Library Online) electronic publication model, formed by a selection of scientific electronic journals, providing free access to the full text and contents of each issue. It also describes in detail the main tools of the Virtual Heath Library - Spain: bibliographic databases (IBECS, MEDLINE, LILACS...), the health terminology "DeCS - Health Sciences Descriptors", the health information locator "LIS-Spain: healthy sites" and the project called "Full text monographs \& bulletins".

Key Words: Virtual Health Library, SciELO, electronic publishing, DeCS, LIS-Spain: healthy sites

\section{ORÍGENES DEL PROYECTO BVS}

El Proyecto Biblioteca Virtual en Salud surge a partir de la preocupación de organismos públicos nacionales e internacionales por ofrecer desde Internet una información científica de calidad garantizada. En 1998, la Organización Panamericana de la Salud (OPS/OMS), se compromete a articular políticas y planes para desarrollar la Biblioteca Virtual en

Correspondencia:

Jorge Veiga del Cabo

Biblioteca Nacional de Ciencias de la Salud

Instituto Carlos III

E-mail: jveiga@isciii.es
Salud (BVS). Para ello se designa al Centro Latinoamericano y del Caribe de Información en Ciencias de la Salud (BIREME/OPS/OMS), como centro coordinador del desarrollo del proyecto BVS.

La Biblioteca Virtual en Salud, fue concebida como una fuente de homogenización, acceso y distribución de la información científico técnica de salud, empleando patrones de selección y estableciendo criterios de rigor y calidad en fuentes y contenido. Para garantizar esta calidad, primero, se aplican una pautas comunes en cuanto al diseño de portales definidas por BIREME/OPS/OMS; y segundo, cada centro coordinador, establece unos criterios de selección para la inclusión de nuevas fuentes de información en cada uno de los portales BVS. 


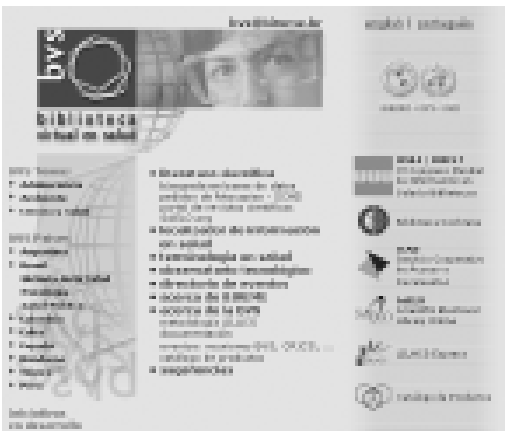

En la actualidad existen más de 20 países iberoamericanos en este proyecto, con casi medio centenar de Bibliotecas Virtuales en Salud temáticas y nacionales, siguiendo unas pautas y metodología comunes, lo que facilita enormemente la navegación del usuario por los diferentes portales. Todas ellas funcionan en red compartiendo diferentes servicios, como el acceso a diferentes bases de datos bibliográficas o el tesauro DeCS.

\section{LA BVS-ESPAÑA}

La OPS/OMS es la organización que escoge al centro coordinador del proyecto en cada país, un centro que tenga la capacidad y los medios para poner en marcha el portal y mantenerlo. Desde el año 1999, El centro coordinador de la Biblioteca Virtual en Salud de España (BVS-España) es la Biblioteca Nacional de Ciencias de la Salud (BNCS).

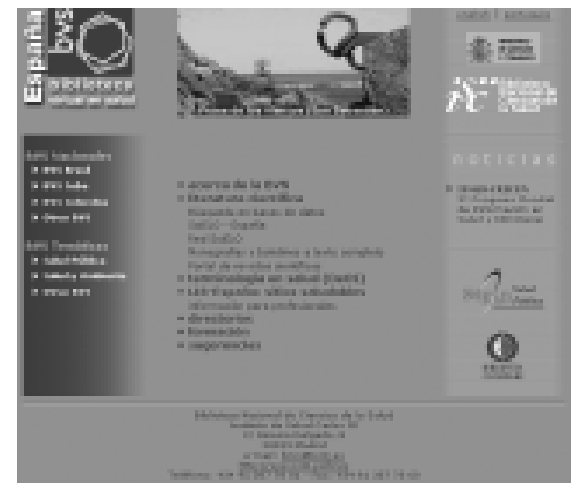

La BVS España cuenta con los siguientes contenidos:

\section{Bases de datos bibliográficas:}

Entre las bases de datos de ámbito nacional, la BVS cuenta con el Índice Bibliográfico Español en Ciencias de la Salud (IBECS). Esta base de datos recoge referencias de artículos de revistas científico sanitarias editadas en España e incluye contenidos de las diferentes ramas de las ciencias de la salud tales como Medicina (incluyendo Salud Pública, Epidemiología y Administración Sanitaria), Farmacia, Veterinaria, Psicología, Odontología y Enfermería. Para garantizar la calidad de los contenidos, cuenta con un Comité Técnico encargado de establecer los criterios de evaluación. Estos criterios contemplan cuarenta y cuatro ítems para evaluar el contenido y rigor científico de las publicaciones, la presentación y la difusión, entre otros factores.

Además de IBECS, la BVS-España permite el acceso a otras bases de datos nacionales e internacionales, como BDIE, MEDLINE o LILACS. Todas ellas utilizan los mismo formularios de búsqueda, lo que facilita en gran medida la localización del información por parte del usuario.

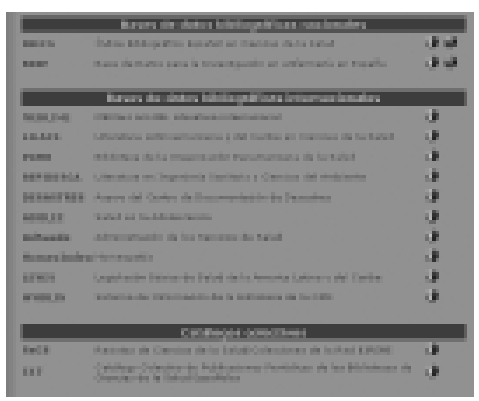

Las bases de datos que conforman la Biblioteca Virtual en Salud, además de ofrecer referencias de artículos, proporcionan conexiones con fuentes externas, como SciELO, proporcionando el acceso al artículo a texto completo, o el directorio de revistas, ofreciendo información sobre la publicación que edita el artículo.

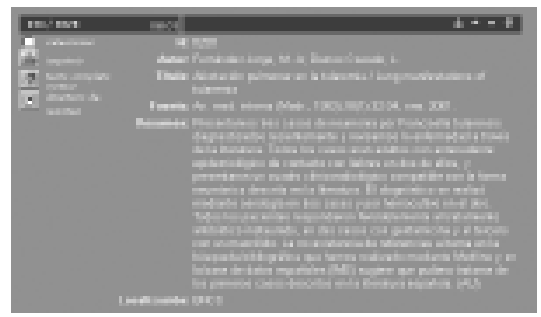

\section{Proyecto DeCS, Descriptores en Ciencias de la Salud.}

En el año 1960, la National Library of Medicine, creó el Tesauro MeSH, Medical Subjet Heading, como vocabulario base para la indización de la base de datos MEDLINE.

En el 1965, BIREME/OPS/OMS comenzó con el proyecto DeCS, Descriptores en Ciencias de la Salud, 
tesauro trilingüe traducción del MeSH al Español y portugués.

Más de 30 años después, el DeCS se convertiría en el tesauro base para la indización de las diferentes herramientas contenidas en la BVS.

España participa en el proyecto DeCS, colaborando en la revisión de los términos en español.

En la actualidad se está trabajando en un metatesauro, Unified Medical Language System (UMLS), que recogerá terminología correspondiente al área de las ciencias de la salud, en múltiples lenguas.

\section{Proyecto Bib-Gen}

Otro de los proyectos a los que se puede acceder a través de la BVS-España es el proyecto Bib-Gen, que tiene como función principal el análisis de las habilidades, conocimientos y motivación necesarios para que los documentalistas y bibliotecarios en ciencias de la salud, accedan a datos del Genoma Humano de utilidad en la práctica de los investigadores y profesionales sanitarios. Para ello, se promueve entre los bibliotecarios y documentalistas que trabajan en Instituciones de Ciencias de la Salud, tanto los conocimientos y habilidades necesarios para acceder a las bases de datos, como los programas empleados para el análisis de secuencias y las herramientas que actualmente se emplean para obtener y procesar la información sobre el Genoma Humano.

\section{SciELO, Scientific Electronic Library Online}

El proyecto SciELO España, constituye uno de los principales contenidos de la BVS-España. Es un modelo de publicación electrónica, que proporciona el acceso al texto completo de una colección de revistas editadas en España del área de las Ciencias de la Salud, seleccionadas de acuerdo a unos criterios de calidad preestablecidos.

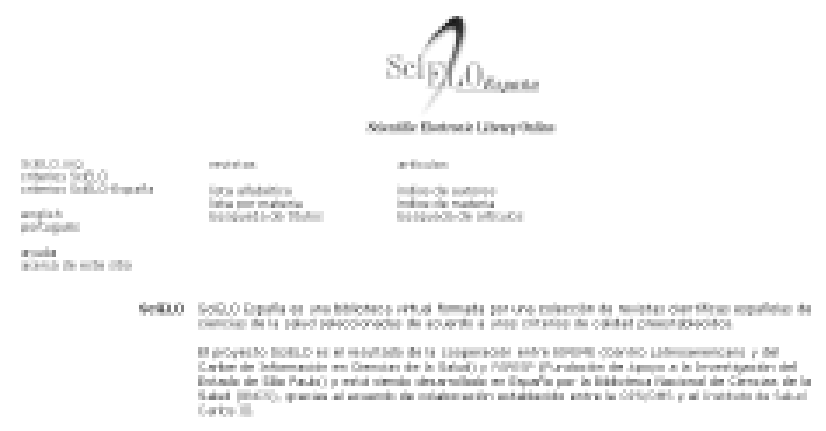

En la actualidad proporciona el acceso al texto completo a 1968 artículos de 13 revistas, entre las que se encuentran Educación Médica, Medicina
Familiar y Comunitaria, Gaceta Sanitaria y Revista Española de Salud Pública.

SciELO proporciona el acceso al resumen y texto completo de cada artículo en formato HTML, así como la opción de su descarga en formato PDF. Ofrece además la posibilidad de guardar las tablas, gráficos e imágenes de modo independiente.

El modelo de Publicación electrónica SciELO, tiene como principal objetivo contribuir al desarrollo de la investigación, mejorando y ampliando los medios de difusión, publicación y evaluación de resultados, creando para ello una red de bibliotecas formadas por colecciones nacionales y temáticas de publicaciones periódicas científicas disponibles a texto completo en Internet. Esta red de bibliotecas se denomina Red SciELO.

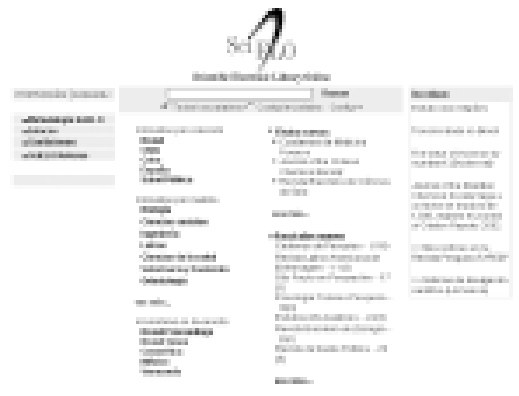

En la actualidad, la Red SciELO permite el acceso al texto completo de 179 revistas a texto completo, perteneciente a 17 materias. Los países que alimentan esta red de bibliotecas de un modo regular son Brasil, Chile, Cuba y España, encontrándose actualmente en desarrollo el proyecto SciELO en países como México, Costa Rica y Venezuela.

Entre los diferentes Modelos de Publicación electrónica, SciELO Brasil es el portal más desarrollado. SciELO Brasil contiene un módulo de estadísticas que proporciona información relativa al factor de impacto de las revistas que lo componen, el índice de inmediatez, y otros datos de interés. En abril de 2004, se espera que este módulo de estadísticas se encuentre también operativo en SciELO España.

SciELO Brasil, ofrece además, conexiones con fuentes externas como MEDLINE, LILACS o Curriculum Lattes.

La Revista Nature, publicó en octubre de 2003 un artículo sobre el proyecto SciELO. En él se explicaba como habían encontrado 5 publicaciones brasileñas indexadas por el ISI desde al menos 5 años, y disponibles en SciELO desde al menos 2 años. Los factores de impacto de estas publicaciones se incre- 


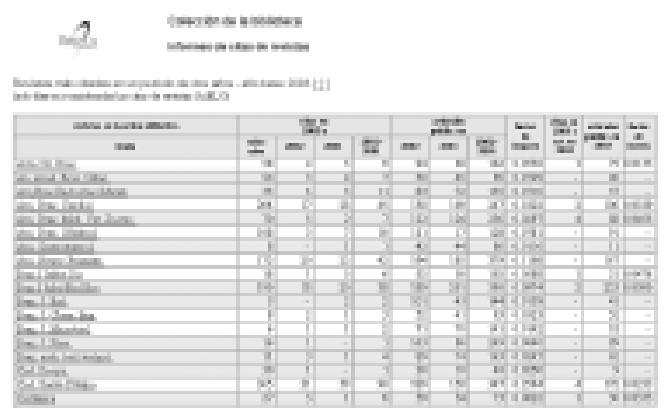

mentaron en más del doble desde sus incorporaciones al modelo de publicación electrónica SciELO. (Alonso, W.J, Fernández-Juricic, E. Regional network raises profile of local journals. Nature 2003 Jan 31; 415: 471-472.)

\section{LIS-España: Sitios Saludables}

El Localizador de Información en Salud, es un buscador especializado en sitios del área de las ciencias de la salud seleccionados mediante criterios de calidad. Estos criterios evalúan cada fuente, teniendo en cuenta el rigor científico del contenido de la página, el acceso, y la autoría entre otros. Ofrece una descripción del contenido del sitio, idioma y su enlace a Internet.

Los formularios de búsqueda de esta herramienta son los mismos que utilizan las Bases de datos contenidas en la BVS y el modelo de publicación electrónica SciELO. Además, permite acceder a la información mediante un índice temático.

\section{Monografías y Boletines a Texto completo}

Es la única herramienta de la BVS-España que se encarga de difundir libros. Ofrece acceso a monografías y boletines a texto completo, en formato PDF. Todos estos documentos cuentan con marcadores para facilitar la navegación por los contenidos de cada obra.

Para facilitar el acceso a cada una de las fuentes, esta herramienta posee un formulario de búsqueda libre, y un directorio donde se recogen todas las obras ordenadas por materia: enfermería, epidemiología, genética y genómica, evaluación de tecnologías sanitarias, patologías y diferentes especialidades médicas.

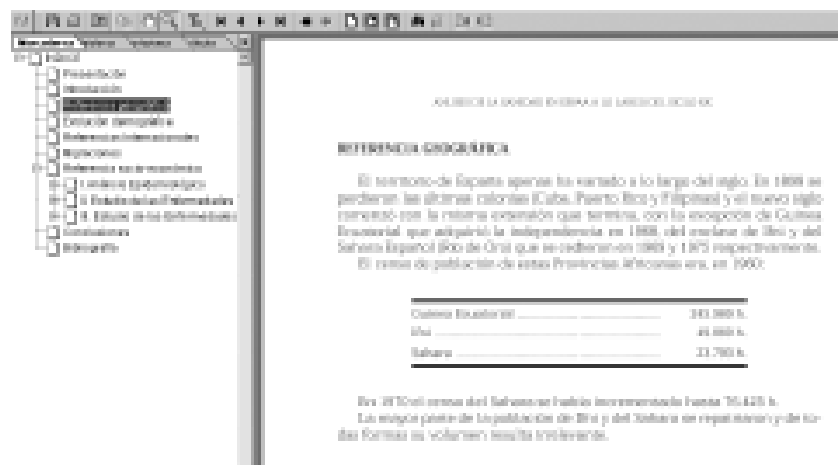

BIBLIOGRAFÍA (consultada)

1. Bojo Canales, Cristina; JAÉN CASQUERO, $\mathbf{M}^{\mathrm{a}}$ Belén. Principales bases de datos en la Biblioteca Virtual en Salud. Libro de Comunicaciones del VI Congreso Nacional de Informática de la Salud. Madrid, 2-4 de abril de 2003. Madrid: Sociedad Española de Informática de la Salud, 2003. pp: 8186. ISBN: 84-930487-6-3

2. Jiménez Planet, Virginia, Jaén Casquero, $\mathbf{M}^{\mathrm{a}}$ Belén. Presentación del nuevo Localizador de Información en Salud: LIS-España. Libro de Comunicaciones del VI Congreso Nacional de Informática de la Salud. Madrid, 2-4 de abril de 2003. Madrid: Sociedad Española de Informática de la Salud, 2003. pp: 35-40. ISBN: 84-930487-6-3

3. Veiga de Cabo, Jorge. El Índice Bibliográfico Español de Ciencias de la Salud. Cooperación con Latinoamérica. Rev. Esp. Salud Publica, Sept./Oct. 1999, vol.73, no.5, p.529-532. ISSN 1135-5727.

4. Veiga de Cabo, Jorge, Martin Pastor, Belén, Calvo Tello, María et al. El modelo SciELO y su contribución a la difusión de las revistas de ciencias de la salud españolas. RCOE, ene.-feb. 2003, vol.8, no.1, p.67-72. ISSN 1138-123X. 